\title{
A Model of Medical Reception - the Planning and Control of Multiple Task Work
}

\author{
BECKY HILL, JOHN LONG, WALTER SMITH AND ANDY WHITEFIELD \\ Ergonomics Unit, University College London \\ 26 Bedford Way, London \\ WC1H 0AP \\ Tel: +44713877050 \\ Fax: +44 715801100 \\ E-mail: ucjtebh@ucl.ac.uk
}

\begin{abstract}
SUMMARY
This research follows in the Broadbent tradition of constructing a general theory to solve applied problems. The paper reports part of wider research intended to construct a framework for modelling the planning and control of multiple task work. The function of the framework is to support the solution of design problems associated with the performance of interactive planning and control worksystems. The study reported here applied an initial version of the framework, based on a study of secretarial office administration, to construct a model of medical reception work. An observational study collected protocol data concerning a medical reception worksystem - comprising two receptionists and various office devices; its domain of application - comprising doctors, patients, their appointments, medical records etc.; and its performance, comprising the quality of medical reception work and the costs to the receptionists of performing that work. The data were then used in conjunction with the planning and control framework to construct the model of medical reception. The model includes physical and abstract structures and behaviours of the medical reception worksystem, as well as physical and abstract objects of its domain of application. Of particular interest for the solution of design problems is the model's differentiation of plan types into task plans, procedure plans, and activity plans. These plan types were used to extend the framework for the planning and control of multiple task work. The paper concludes with a brief illustration of how the model of medical reception might be used to support the solution of interactive worksystem design problems as they relate to performance. Future work to develop further the framework of planning and control of multiple task work and its application are discussed.
\end{abstract}




\section{INTRODUCTION}

\subsection{Applying Research Knowledge}

How to ensure the applicability of the knowledge generated by its research is a persistent problem for many disciplines, including cognitive psychology. Donald Broadbent was much concerned by this problem; and in particular by the relation of cognitive theory and application in psychology (1971a). His position on the relation, however, was clear and often stated. First, he held that problems should be drawn from real and practical situations, in which human beings naturally perform. Second, he held that the best contribution to practical problems is to produce a general theory (1971b). Third, he held that the most valid general theory is one which works, that is one from which a solution to an applied problem may be derived (1971a). The work reported in this paper follows directly in the Broadbent tradition: addressing a practical situation medical reception; attempting to construct a general theory - of planning and control of multiple tasks; and with an aim of solving, by means of the theory, an applied problem designing user interfaces for interactive computer systems. The work, however, has also sought to advance that tradition by attempting to develop a theory which ensures better support for the solution of applied problems. The advance is based on three requirements. First, the applied problem, not just the theory, needs a technical expression. Second, the theory needs to address all aspects of the applied problem, not just the human behaviours associated with it. Third, the form and granularity of the theory need to be commensurate with the applied problem. In attempting to meet these requirements, the research reported here has sought to construct a theory more fit for the purpose of solving applied problems.

As indicated, one possible application of cognitive psychology research is to support the solution of design problems of user interfaces associated with the performance of interactive computer systems. The work reported here was part of a project which aimed to produce a design-oriented framework for modelling the planning and control behaviours of people and machines performing multiple task work. That is, the research was concerned with modelling particular cognitive behaviours of users interacting with machines effectively, such as to support the application of the model to the design of interactive computer systems involving those behaviours. The research investigated the planning and control of multiple task work for three types of administrative work: secretarial office administration; medical reception; and legal service provision. The investigation reported here aimed to extend to medical reception the framework previously developed for secretarial office administration, and to develop the framework to include any additional generic planning and control behaviours identified in the medical reception worksystem. 
The remainder of Section 1 provides background information about medical reception and presents the framework. Section 2 describes the particular medical reception worksystem studied and the data collection and analysis methods employed. Section 3 presents the resulting model of planning and control in medical reception, including the different types of plan used by the medical reception worksystem. The extension of the framework for the planning and control of multiple task work is also described. Section 4 discusses how the model might be used in worksystem design, and Section 5 offers a summary and some conclusions.

\subsection{Medical Reception (in the UK)}

Informally, medical reception worksystems can be identified as those interactive systems, comprising combinations of people and office devices, which support the effective interaction between medical practitioners and their patients in medical general practices.

Jeffreys and Sachs (1983) described the emergence of medical reception worksystems in the UK. In 1966, there was a boost to the employment of receptionists and secretaries, because the Family Doctors Charter gave provision for GPs to reclaim 70\% of the salaries paid to their staff. Closely related to the increasing employment of receptionists was the growth in the use of appointment systems in general practice, as an appointment system could not be implemented without the employment of receptionist staff. By 1981, over $70 \%$ of medical general practitioners in the UK employed receptionists and operated appointment systems (Drury, 1981).

General practices have begun to computerise their administration, although by the early 1980s the number was still small (Jeffreys and Sachs, 1983). As part of its recent National Health Service reforms, the UK government has attempted to increase the financial and managerial autonomy of general practitioners. This increase has included a scheme for partial reimbursement of computer costs to increase computerisation.

Medical reception work is therefore an area with a significant number of potential users, with a currently low level of computerisation, and with political and financial pressures to increase computerisation. As such, it presents an example of what might be described as an emerging Human Computer Interaction (HCI) design problem. Following the approach of Dowell and Long (1989), the medical reception HCI design problem might be stated as: to specify the structures and behaviours of a human-computer interactive medical reception worksystem which will carry out work in the domain of medical reception to a desired level of medical reception performance. 


\subsection{The Framework}

Prior to the present investigation of medical reception work, the research had constructed a framework for modelling the planning and control of multiple task work (the PCMT framework), based on the work of secretarial office administration. The full details of how this framework was derived and the model constructed is reported in Smith, Hill, Long and Whitefield (1993).

The PCMT framework constituted a domain-independent expression of how worksystems perform the planning and control of multiple task work. The framework was to be further developed by studies of medical reception work and of solicitors' work. It provided a structure within which to model these different types of administrative work. This section introduces the PCMT framework, which is used in Section 3 to model the data obtained in the medical reception study.

\section{INSERT FIGURE 1 ABOUT HERE}

The PCMT framework is represented diagramatically in Figure 1. It is based on Dowell and Long's (1989) conception for an engineering discipline of HCI which expresses the HCI general design problem. The conception makes a fundamental distinction between an interactive worksystem, comprising one or more users and computers, and its domain of application, comprising the transformations carried out by the worksystem which constitute its work. The effectiveness with which work is carried out is expressed by the concept of performance which can be defined as a function of two factors: the quality of the product (i.e. how well the final state of the domain compares with its desired state as specified in the work goal); and the incurred resource costs (i.e. the resources required by the worksystem in accomplishing the work). Design problems are expressed as unacceptable levels of performance.

In the PCMT framework, a domain of application (or work domain) is made up of objects, which may be abstract or physical. Objects are defined by their attributes, which have values. The attribute values of an object may be related to the attribute values of one or more other objects. The state of an object at any time is determined by the values of its attributes. The worksystem performs work by changing the state of domain objects (i.e. by transforming their attribute values) towards their desired states as specified in the work goal.

To illustrate the application of the framework to medical reception work, the provision of support for medical case objects (i.e. patients consulting with medical practitioners) occurs by manipulating the attribute values of other objects such as patients, doctors, 
prescriptions, medical record envelopes, etc. An example attribute of a patient object is appointment-time. Initially the appointment-time attribute has a value of none as the patient does not have an appointment booked at the surgery. Upon the worksystem booking an appointment for the patient the value of the appointment-time attribute will be transformed to the value of the time of the allocated appointment slot, for example 2 p.m..

The medical reception domain is an instance of multiple task work, since support is provided concurrently for multiple ongoing and temporally overlapping medical cases (i.e. for many patients together). In the framework, performance expresses the quality of the multiple tasks, that is how well they are carried out, and the costs of their planning and control. Performance in medical reception concerns the quality of the medical cases supported and the costs to the medical reception worksystem of providing that support.

The PCMT framework defines a number of worksystem structures for the planning and control of multiple task work. These structures are expressed at both abstract and physical levels of description. Firstly, the framework describes the worksystem's cognitive structures. These structures comprise four processes (planning, controlling, perceiving and executing processes), and two representational structures (plans and knowledge-of-tasks). The four processes support the behaviours of planning, control, perception and execution respectively. The full argument for this set of structures can be found elsewhere (Smith, Hill, Long and Whitefield, 1992) but can be summarised as follows.

Influenced by Newell and Simon (1972), much planning research in cognitive science and artificial intelligence has tended to view plans as complete and fully-elaborated behaviour sequences which ensure task goal achievement. This view has been undermined by research into planning in HCI. The behaviours of users who are part of worksystems, it has been argued, cannot be regarded entirely as the output of executable plans (e.g., Suchman, 1987; Larkin, 1989; Payne, 1991) - rather they are often, at least partly, direct responses to the task environment. Within this perspective, plans need not be complete and fully-elaborated, but rather they may be partial (in the sense that they may specify only some of the behaviours to be implemented) and/or general (in the sense that some behaviours may be specified only generally and not at a level that is executable). Such plans might be more generally viewed as 'resources' for guiding behaviour (Suchman, 1987). Furthermore, if a plan is regarded as a resource to guide behaviour it is no longer necessary that it be limited to specifying behaviours, rather it might instead specify required states of the task or conditions of the environment. Plans 
which serve as resources for guiding behaviour, rather than as specifications of complete and fully-elaborated behaviour sequences, cannot ensure that goals will be achieved.

This also undermines the assumption that perception precedes planning which precedes execution. Ambros-Ingerson (1986) argued that all planning can precede execution only when:

1. The task environment is static - relevant changes in the task environment do not occur after the plan is complete; and

2. The task environment is simple enough to be practically modelled - the consequence of behaviours can be predicted sufficiently well to generate a complete and fully-elaborated behaviour sequence; and

3. The task environment is known - the planner's knowledge of the task environment can be complete before planning commences.

Most task environments studied by HCI researchers do not embody these assumptions (Young and Simon, 1987). In direct contrast, they are usually dynamic, complex and partly unknown by the planner (e.g., Hollnagel, Mancini and Woods, 1988). Execution behaviours in worksystem task environments are required to commence before plans are complete and fully-elaborated, and therefore the perception, execution and planning behaviours must be temporally interleaved - having no necessarily fixed order in which to be performed.

When performing a task, a system has to exercise control; that is, it has to select the next behaviour to be carried out at each moment (e.g., Hayes-Roth, 1985). For a system which constructs complete and fully-elaborated plans, controlling is a simple process of selecting behaviours according to the plan and initiating their execution. However, for worksystems, which employ plans as resources to guide behaviour, some more complex control behaviour is required to select execution behaviours over time - since the selection is constrained by, rather than specified by, the plan. Furthermore, if a worksystem interleaves execution behaviours with planning and perception behaviours, controlled sequencing of these behaviours is also required.

Based on the preceeding arguments, the PCMT framework describes the worksystems cognitive structures for planning and control as follows:

At the First (abstract) level of description, Plans are specifications of required transformations of domain objects and/or of required behaviours. They may be partial (in the sense that they may specify only some of the behaviours or transformations), and they may be general (in the sense that some behaviours or transformations may be 
specified only generally and not at a level that is directly executable). In the case of medical reception, for example, planning behaviours specify the required transformations and/or behaviours to support medical case objects, such as specifiying a required transformation of the location of a repeat prescription or, specifying the sequence of worksystem behaviours that will provide a patient with an appointment.

Perception and execution behaviours are, respectively, those whereby the worksystem acquires information about the domain objects and those whereby it provides the required support for those objects. In the case of medical reception, perception behaviours acquire information about doctors, patients, and other domain objects, such as their location and their requirements. This information appears in the knowledge-oftasks representation. Execution behaviours to transform domain objects would include informing patients of their appointment times and writing repeat prescriptions.

Control behaviours entail deciding which behaviour to carry out next, but are more than the process of reading off the next behaviour from a complete and fully-elaborated plan. In the case of medical reception, control behaviours might decide, for example, to perform next the execution behaviours of arranging an appointment for Patient 1 , in preference to the perception behaviours of answering the telephone, or to planning behaviours of specifying how to complete a repeat prescription for Patient 2.

The second level of description of planning and control structures is physical, wherein the framework describes the distribution of the abstract cognitive structures across the physically separate user and devices of particular worksystems. The framework therefore allows the construction of alternative models of the distribution of cognitive structures across the user and devices ${ }^{1}$, and thus it supports reasoning about allocation of function. In medical reception, the physical worksystem is the receptionist plus devices such as an appointment book, telephone and prescription filing system. This notion of worksystem is slightly wider than that in Dowell and Long's (1989) conception, and is used here for the analysis of to-be-computerised systems.

The outline of the PCMT framework, including its domain of application, its worksystem and its performance, is now complete. The components of the framework have been informally exemplified for medical reception work. This framework has been used to analyse the medical reception data of the study whose collection is described in the next section.

\footnotetext{
${ }^{1}$ It is assumed here (and elsewhere in the paper) that both natural and artificial systems can have cognitive structures.
} 


\section{AN OBSERVATIONAL STUDY OF MEDICAL RECEPTION}

This section describes the observational study of a medical reception worksystem. The study was intended to provide empirical support for the construction of a model of PCMT for medical reception, and in addition to develop further the PCMT framework to include planning and control of multiple tasks in a work domain additional to that of secretarial office administration.

\subsection{The Nature of Medical Reception}

The medical reception domain is described in terms of the PCMT framework in Section 3. In this section, the concern is with an informal description of medical reception, sufficient to justify the selection of data collection techniques.

Medical reception involves interaction with, and manipulation of, a range of physical objects, both human and non-human. These objects include patients; doctors; nurses; prescriptions; medical record envelopes; test results; and so on. But the particular physical objects involved in any patient-doctor consultation will vary both within and between patients; for example, the same patient may see different doctors, with or without different test results, X-rays, prescriptions, etc. One means to express the goal of transforming these physical objects is to postulate an abstract medical case object for each patient. The goal of the medical reception worksystem is to support this abstract medical case object effectively by ensuring that the patient can obtain the most suitable appointments and that the correct test results, medical record envelope and so on are available as needed.

This view of medical reception offers a number of characteristics which may differ from other domains. For example, receptionists deal with many patients, but for much of the time the receptionists repeatedly perform a relatively small number of tasks for those patients (e.g. make appointments, hand out test results, take repeat prescription requests). Medical reception work, therefore, seems to involve well-defined, routine subtasks, which may be of variable durations (since consultations may occur in one day or over several weeks). Further, the domain involves a high frequency of autonomous events, i.e. task-relevant events which occur independently of any worksystem behaviour, for example, the arrival of a patient or an incoming telephone call.

This informal view of medical reception requires that the data collection techniques be capable of capturing: how the medical reception worksystem interacts with a range of physical objects; how changes in those objects occur (including autonomous events); how effective are these changes; and how more abstract elements of medical reception (such as appointment suitability) are addressed. 


\subsection{The Medical Reception Worksystem}

In choosing a medical reception worksystem for study, two factors had to be respected. Medical reception work is considered confidential; i.e. patients expect their interaction with the receptionists to be as confidential as their interaction with the Doctors at the surgery. Further, the work of the medical receptionists varies with the size of the surgery, and the existence, or not, of an appointment-booking system.

For example, in a small practice (comprising one or two Doctors) during surgeries (i.e. when the doctors are seeing patients), the receptionist attends to the reception desks. If there is no appointment system, the receptionist takes charge of the work which, at group practices, is undertaken by nurses, record clerks, secretaries or a practice manager, e.g. completing vaccination forms; supplying of syringes, dressings, stationery; typing letters for hospital admissions; arranging ambulance transport. However, in a Group practice (comprising three or more doctors) most of the receptionists' time is spent at the reception desk, where they deal with: requests for surgery appointments and home visits, either by phone or in person; patients who turn up with or without appointments; telephone requests to speak to doctors and other medical health workers; registration of new patients; requests for repeat prescriptions; and a myriad of patient enquiries and complaints.

Thus, the medical reception worksystem chosen for study needed to allow access to the worksystem in such a way that the patients' privacy was maintained, and preferably be situated within a surgery where an appointment-booking system was in operation. This latter constraint was to ensure that the work of the MR worksystem was restricted to supporting the consultations between the patients and their doctors, and excluded work carried out by other staff under different circumstances (and which might therefore be considered as outside the domain of medical reception).

The medical reception worksystem chosen for this study supported the provision of medical care in a general practice with four doctors and two nurses. This worksystem was physically divided into two different workstations, with two receptionists, one working from a 'front desk' and one from a 'back desk'. A plan view is depicted in Figure 2. The front desk workstation comprised a receptionist and devices, such as a telephone and an appointments book. The back desk workstation comprised a second receptionist and devices, such as a prescription book, telephone and a computerised database. The front desk was positioned by a hatch through which the receptionist interacted with patients arriving at the surgery. The position of the hatch ensured that the privacy of the patients could be respected as it was possible to record the patients' speech without them 
being seen. Under instruction from the receptionist, patients passed from the hatch to a waiting room before consulting with a medical practitioner. Activities performed at the front desk workstation included: booking appointments; registering new patients; and dealing with patients arriving and leaving the surgery. Activities performed at the back desk workstation included: notifying patients of their test results; preparing (and updating) medical notes for the medical practitioners; and preparing the repeat prescriptions.

\section{INSERT FIG 2 ABOUT HERE}

\subsection{Data Collection}

To decide the type of data collection, a preliminary analysis of the medical reception worksystem was carried out. This investigation entailed an informal observation of the worksystem for one day without recording equipment. The preliminary study revealed that the worksystem was very busy and that it would be difficult to ascertain the receptionists' activities by means of unrecorded observations. Sound recording alone would also be unsatisfactory as the important non-verbal behaviours of the receptionist would not be recorded. Therefore, it was decided to use video recording. Video recording would provide a complete representation of the behaviours and work being carried out within the study environment, over an allotted period of time. The video recording would also provide a high quality sound record and would be time-stamped. A video recording is also a permanent record, which can be analysed at length. (Due to the confidentiality of the work of medical reception which required the anonymity of the patients it was not possible to record the incoming speech of a telephone call.)

To obtain an accurate description of the work of the receptionists, video recordings were made, both during and outside surgery hours, for one morning and afternoon, in which time one pair of receptionists was relieved by another. Two video cameras were used simultaneously, one camera focused on the appointment-booking system of the front desk, while the other camera recorded the interactions within the whole reception area, including both desks. At a later date, after initial analysis, an interview was carried out with one receptionist, to obtain clarification of selected details concerning the work. The interview was informal and will not be reported here separately, as the information gathered has been incorporated into the analysis.

\subsection{Data Analysis}

The data collected by video were: 
- The task-related events that occurred e.g. telephone enquiries and patients arriving.

- The receptionists' interactions with their devices e.g. the appointment book and telephone.

- The receptionists' interactions with the doctors.

- The receptionists' interactions with each other.

- The receptionists' interactions with the patients.

Only the two videos recorded in the morning were analysed, because the data gathered therefrom included a sufficient amount of the data types listed above for the required analysis.

The following analysis was carried out on both videos. From the 240 minutes of videorecording, various sequences were selected for analysis, which varied in length between 13 and 62 minutes. This selection was based on the criteria that:

- the observed behaviours were interpretable (including being able to decipher a telephone call without having recorded both sides of the conversation)

- the analysed period appeared to be busy in support of medical cases (and so was presumed to include behaviours of interest)

- the data listed above occurred frequently.

The sequences varied in length because the activities being carried out at the two workstations (front and back desks) had different durations; the front desk activities had shorter durations than the back desk activites, so that althought the sequences analysed varied in length the content of the sequences in terms of numbers of behaviours recorded did not.

The first stage of the analysis was the documentation of behaviours and task-related events, from the sequences, to a level of description considered to be at, or below, that necessary for the identification of medical reception planning and control behaviours. This documentation entailed a first description, recording all verbalisations verbatim², and all non-verbal behaviours of the receptionist and events in chronological sequence. This 'raw protocol' was then further analysed to identify the physical embodiments of the abstract IWS structures and physical domain objects from the PCMT framework. This process was iterative. This further analysis produced an annotated protocol which identified:

- the physical domain objects (e.g., prescription);

- the physical worksystem devices (e.g., telephone1, prescreiption box);

- the behaviours of the receptionist.

\footnotetext{
2 The real names of staff and patients were changed to initials to preserve confidentiality.
} 
Figure 3 shows a section of the annotated protocol from the workstation of the front desk receptionist. (Space limitations preclude reporting the complete set of protocols. ${ }^{3}$ ) The sequences of videotape analysed are all documented to this level of detail.

\section{INSERT FIGURE 3 ABOUT HERE}

From this further analysis, each behaviour in the protocol was then associated with a specific task. For example, in Figure 3 the left hand side number on each line refers to a specific task: Number 1 identifies worksystem behaviours involved in booking an appointment for a patient; Number 2 identifies worksystem behaviours involved in registering a new patient. With very few exceptions, all the behaviours in the protocol were associated with a single task. Some behaviours could not be associated with a specific task, because the protocol contained insufficient data or because they were isolated behaviours relating to tasks which otherwise took place outside the time period recorded or that selected for analysis.

The analysis, therefore, attempted to document relevant observable structures and behaviours of the medical reception worksystem in as much detail as possible, and identified the specific tasks in the medical reception domain to which each behaviour relates. The next section describes the medical reception model constructed from this analysis.

\section{MODEL OF PCMT-MR}

Section 1.3 described the framework of PCMT constructed prior to conducting the observational study outlined in Section 2. Section 3 now describes the model of PCMTMedical Reception (MR $)^{4}$ constructed by using the observations of medical reception tasks and behaviours to instantiate the concepts of the framework.

The modelling of medical reception can be divided into two activities:

- describing the medical reception domain, including all the identified domain objects and their attributes.

- describing the medical reception worksystem, including details of the observed plans.

\footnotetext{
3 However, a complete set of protocols, comprising 20 A4 pages, is available on request from the first author.

4 Strictly speaking, the model of PCMT-MR consists of a PCMT-framework (Section 1.3); an MRframework (comprising the generic aspects of MR); and a PCMT-MR model which represents the instances of the MR domain, worksystem and performance. However, to avoid confusion here, the generic and instance components of PCMT-MR are both considered as the MR model.
} 
Medical reception performance can then be exemplified as the relationship between the domain of medical reception, expressed as task quality and user costs, and the medical reception worksystem.

Figure 4 provides an overview of the model of PCMT-MR which is now described.

\section{INSERT FIGURE 4 ABOUT HERE}

\subsection{Medical Reception Domain}

Based on the PCMT framework, the medical reception domain is expressed as those objects whose transformation constitutes the work of medical reception. In the PCMTMR model, the domain contains multiple medical case objects, each of which itself comprises four objects: a patient object; medical practitioner object(s); diagnosis object(s); and treatment object(s). The medical case objects and their component objects are all abstract. The domain contains in addition physical objects, to be described below.

Figure 4 shows all the attributes associated with the abstract domain objects. Each task constitutes the transformation of a single medical case object with respect to the values of a number of these attributes. The attributes of a medical case object which must be transformed are: appointment suitability for the patient; appointment suitability for the medical practitioner; treatment support accuracy; and diagnosis support accuracy. In order to transform the medical case object attributes, the attributes of the componentobjects must be transformed. The transformations of the abstract object attributes result from the manipulation of the physical domain objects by the worksystem. Detailed examples of these attribute transformations are provided later in this section.

Figure 4 shows all the types of physical domain object (but not their attributes) in the PCMT-MR model. Included are all the physical objects which were identified by the protocol analysis and which were assigned to particular medical cases. A small number of physical objects in the protocol were not assignable to medical cases. The reason was incomplete information available within the protocol about the particular medical case to which they related. For example, during the protocol the receptionist may open a letter, but then simply leave it on the desk to be dealt with later, without saying anything about it. That letter (whether it be some test results or a request for an appointment) cannot be assigned to a particular medical case. If the protocol covered a longer period of time (during which the receptionist dealt with the letter) or if it contained more detail (e.g. if its contents could be read from the video recording), then the letter would be assignable to its appropriate medical case. Ideally the data collection would have covered a longer 
period of time so that details could be gathered about tasks which have a longer duration than one day. Further Studies of other medical reception domains would require the data collection to cover a long period of time to gather a richer set of data.

The required transformation of each medical case object can be divided into a number of sub-transformations concerning particular sets of attributes. The division of the tasks into sub-transformations was consistent across all the tasks, in the sense that a given subtransformation did or could occur for any medical case. Therefore the subtransformations can be considered generic sub-tasks. The generic sub-tasks identified here are shown in Table 1:

\section{INSERT TABLE 1 ABOUT HERE}

Tables 2a-4c describe the transformation of the abstract objects associated with three ${ }^{5}$ of these generic sub-tasks: appointment-booking; repeat prescription preparation; and medical notes for medical practitioners preparation (and updating). However all the subtasks identified within medical reception can be described in the same way in terms of their associated set of domain object transformations, which can be derived from the domain description and the protocol descriptions of the behaviours associated with each generic sub-task.

\section{INSERT TABLES 2a-4c ABOUT HERE}

The tables show (on the left hand side), the attributes of the medical case objects and the sub-objects of patient, medical practitioner, treatment and diagnosis, and (on the right hand side), the attribute value changes for a particular example for each of the sub-tasks. To explain more clearly the transformation of attribute value changes, Tables 2a-2c, which show the attribute value changes associated with an example of appointmentbooking, will now be described in more detail.

For the sub-task of appointment-booking, one of the attributes of the medical case object, which must be transformed, is suitability of appointment for the patient. To transform this attribute, the attribute values of appointment-time and appointment-medical practitioner of the patient sub-object must be transformed. The value of these attributes must then be compared with the patient's attribute values of appointment requirements to assess the suitability of the appointment for the patient, thus transforming this

\footnotetext{
${ }^{5}$ Space precludes documenting all the generic sub-tasks.
} 
attribute of the medical case object from not known to either suitable or unsuitable. For the example shown in Tables 1a-1c, the appointment booked has the desired value for the medical practitioner, that is $\operatorname{Dr} Y$, and the desired time, that is Wednesday. Therefore, the suitability of appointment for the patient attribute value change of the medical case object, would be from not known to suitable.

The transformation of the abstract object attributes takes place as a consequence of the manipulations of the physical domain objects by the medical reception worksystem. To exemplify this relationship between the abstract and physical domain objects, a detailed account of the repeat prescription preparation example, described in Tables 3a-3c, will now be given in terms of the physical domain object attribute transformations.

For the sub-task of repeat prescription preparation, the medical reception worksystem has to support the accuracy of the treatment of a patient by prescription. The physical domain object to be transformed during this sub-task is a prescription. A prescription has attributes of: patient identity; doctor identity; prescribed treatment name; and $\underline{\mathrm{Dr}}$ authorisation. At the start of the sub-task of preparation of repeat prescriptions, the physical prescription object attributes have no values, except for Dr authorisation, which has a value of not authorised. The medical reception worksystem must transform the prescription object by giving values to its attributes as required to support the treatment of the relevant patient. For the case presented in Tables 3a-3c, a patient Mrs $X$ has requested a repeat prescription for treatment $X$ prescribed by Dr Y. Mrs X's doctor is Dr Y. Therefore, to support this particular request the receptionist must transform the attributes of the prescription object from: Patient identity: none - Mrs X; Doctor identity: none - Dr Y; Prescribed treatment name: none - treatment X. These transformations are effected by the receptionist writing the desired attribute values onto the prescription object. The prescription object now requires authorisation by Dr Y. Thus, the receptionist will place the prescription into Dr Y's tray, for Dr Y to sign. When the prescription has been signed by the doctor, the attribute value of Dr authorisation will have been transformed from not authorised to authorised. The prescription is returned to the medical reception worksystem, where it is placed in the prescription box on the front desk, ready for collection by the Patient, Mrs X (see Figure 2).

Attributes may be affordant or dispositional. Affordant attributes are transformed by the worksystem; their transformation constitutes the work performed. Dispositional attributes are relevant to the work (they need to be perceived by the worksystem), but their transformation does not itself constitute work (often dispositional attributes do not change their values). For example, for the sub-task of appointment-booking, one of the affordant attributes for the patient object is appointment time. This attribute initially has 
a value of none, as when the patient requests an appointment, although they may have some desired time for their appointment (appointment-requirements: when - Wednesday), they do not know whether that appointment slot is available. They find out only when the receptionist informs them and records the appointment in the appointment book. Only then is the attribute value of appointment time transformed to the recorded appointment time. However, the attribute of appointment-requirements: when, is dispositional, as the patients' desired appointment time does not change. The receptionist needs to perceive the value of this dispositional attribute, so that they can offer the patient an appointment day which either matches the desired attribute value (Wednesday), or is the most suitable of the available appointments (e.g. Thursday). The attributes marked with an asterisk $\left.{ }^{*}\right)$ in Tables $2 \mathrm{a}-4 \mathrm{c}$ are dispositional.

Associated with the set of identified generic sub-tasks there were a corresponding set of activities. An activity is that set of behaviours which carry out a generic sub-task.

The activities identified in medical reception are shown in Table 5:

\section{INSERT TABLE 5 ABOUT HERE}

The description of the medical reception domain as part of the PCMT-MR model is now complete. The next section describes the second part of the model - the medical reception worksystem.

\subsection{Medical Reception Worksystem}

The expression of the medical reception worksystem in Figure 4 shows the cognitive structures of the PCMT framework (described in Section 1.3). The relationships between the cognitive structures in Figure 4 embody the planning and control behaviours described in Section 1.3 (for more details see Smith et al, 1992b). The framework comprises four processes - planning, controlling, perceiving and executing - and two representations - plans and knowledge-of-tasks. Within medical reception, these cognitive structures were identified as follows.

- A perceiving process by which the receptionist acquires information about the medical cases and updates their knowledge of tasks. For example, perceiving information about when and with whom a patient requires an appointment.

- A planning process which constructs plans, based on knowledge-of-tasks. For example, using information in the knowledge-of-tasks, that a patient requires an 
appointment today, the receptionist will select and mentally mark a possible appointment slot in the task plan i.e. in the appointment book.

- An executing process, which transforms the attribute values of the domain objects directly. For example, informing the doctor of his appointment schedule for the morning transforms the medical practitioner object attribute of knowledge of appointment from a value of not informed to informed.

- A controlling process, which decides which of the other processes should be carried out next, based on the plans and knowledge-of-tasks. For example, having information about a patient's desired doctor for an appointment, and having updated the knowledge-of-tasks - if the desired appointment time is still not known, and the patient is readily accessible, then the controlling process may direct the perceiving process to elicit the required information from the patient, in preference to directing the perceiving process to answer a telephone ringing within the worksystem.

- A plan representation structure which embodies three plan types: the task plan; activity plan; and procedure plan.

- A knowledge-of-tasks structure which represents knowledge of relevant aspects of the work domain. For example, the required drug name for a particular repeat prescription.

These representations and processes are distributed across the receptionist and physically distinct worksystem devices. For example, the appointment book embodies part of the task plan as it contains information about the identity of the patient, the doctor, and the time and day of an appointment. The information is specific to a particular medical case, and thus to a specific task. The receptionists' mental markers (see below for details) also embody part of the task plan, as the mental markers are mental representations of available appointment times with particular doctors. These times are specific to particular medical cases and therefore to specific tasks. The telephone and the receptionist together embody a perceiving process of the worksystem. Thus as a perceiving device the telephone is activated by someone trying to contact the worksystem and therefore rings. The receptionist must then pick up the receiver of the telephone in order to complete the perceiving process of the worksystem of discovering the nature of the call and updating the knowledge-of-tasks representation, with information perceived via the telephone.

The plan representation structure in Figure 4 has been 'opened-up' to show the different types of plan identified in this study. These types will now be described in detail.

\subsubsection{Plans and planning in the medical reception worksystem}


In the PCMT-MR model, plans are representations of how tasks are to be accomplished, specified to some level of completeness, some level of detail and in some format. In the study of medical reception, it was possible to identify three different types of plan employed by the worksystem. This section describes these three types in turn, and shows how they were interpreted as instances of three general types of plan: a task plan; an activity plan; and a procedure plan.

\section{The Task Plan}

The receptionists used two appointment books (one for doctors and one for nurses) to represent and record details of patient appointments. Figure 5 schematically depicts all the information represented in the appointment book for doctors: names of patients occupying particular appointment slots; whether or not the patient had entered the waiting room; slots which were still available; slots which the medical practitioners wanted to be left open; slots which could be used in emergencies. The receptionists also used what can be called 'mental markers'; that is, they made mental notes of temporary significant appointment slots, such as the next available appointment of a particular medical practitioner or a slot which was in the process of being offered to a patient, but not yet accepted.

\section{INSERT FIGURE 5 ABOUT HERE}

In the present analysis, the appointment books, plus the associated mental markers, were regarded as plans of the medical reception worksystem, because they guided its behaviour. For example, they represented the patients whose medical notes needed to be prepared for the doctor, and the patients who should be allowed into the waiting-room. In terms of the PCMT-MR model, the appointment books were plans which represented information about domain object attribute values. Specifically, they represented information about the patient object attributes of appointment-time and appointmentmedical practitioner, and the medical practitioner object attribute of availability (see Figure 4).

The information represented in the appointment books was specific to particular objects, i.e. patients and medical practitioners in the medical reception domain. The information was therefore specific to particular tasks, i.e. transformations of medical cases. The appointment books, with associated mental markers, were therefore identified as instances of a generic type of plan - the task plan. In general, task plans are specifications of either behaviours or domain object transformations relating to specific task instances. The appointment books were partial task plans, because they only contain information about the patient and medical practitioner objects of a medical case, and not the 
treatment and diagnosis objects. Further, they contained specifications of only domain object transformations and not of behaviours.

The task plan, observed in the form of patient appointment books, supported the effective performance of the multiple tasks within medical reception work by:

- giving guidance for the performance of behaviours relating to specific tasks; e.g. whether to admit Patient P1 to the waiting room, preparing medical notes for Patient P2;

- co-ordinating different tasks; e.g. ensuring that appointments were unique for each task.

\section{The Activity Plan}

As described earlier, the medical reception worksystem carried out a number of different activities, e.g. booking appointments, preparing medical notes. From the video recording and interview, it was possible to identify that the receptionists had a shared daily schedule of activities, mentally represented, to be carried out by the front and back desk receptionists. Figure 6 shows the complete activity schedule of the observed medical reception worksystem on the day of recording. This schedule was not rigidly adhered to as many activities, such as notification of test results, were carried out in direct response to autonomous events such as patients telephoning the surgery.

\section{INSERT FIGURE 6 ABOUT HERE}

The information represented in the activity schedule was specific to the carrying out of particular activities, as opposed to particular tasks. The activity schedule was, therefore, identified as an instance of a generic type of plan - the activity plan. In general, activity plans are specifications of sequences of activities to be carried out; where each activity is a set of behaviours relating to a particular generic sub-task of the domain (see Section 3.1).

The activity plan, observed in the form of a (mentally represented) daily schedule of activities, contributed to the effective performance of tasks by:

- supporting extensive sharing of effort across separate tasks; e.g. when carrying out the activity of preparing repeat prescriptions, all of the medical notes for the patients requiring repeat prescriptions would be collected together at one time.

- co-ordinating the activities with the task-relevant changes in the domain; e.g. the activity of preparing repeat prescriptions was carried out during surgery hours, so that the prescriptions were ready for the doctors to verify and sign when the surgeries finished.

\section{The Procedure Plan}


From analysis of the video recordings, supported by interviews, it was possible to identify that the receptionist performed well-established sequences of behaviours when carrying out a particular activity. Thus, the receptionists had mental routine structures, which represented information about behaviours and their contingencies for particular activities. These routine structures were identified as instances of a generic type of plan the procedure plan. In general, a procedure plan specifies an efficient sequence of behaviours, and their contingencies, for carrying out a particular activity which relates to a generic sub-task of the domain (see Section 3.1).

There are two stages in specifying a procedure plan for a particular activity. The first stage requires the informal documentation of the procedure for carrying out a particular activity, based on the analysis of the protocols. The second stage requires a more precise description to identify clearly the worksystem behaviours and their associated domain object transformations, and to represent this information in a more structured manner.

As an illustration, the procedure plan for booking appointments is now described in detail. Informal descriptions of the other activities identified within medical reception appear in Appendix 1.

\section{INSERT FIGURE 7 ABOUT HERE}

Figure 7 shows a flow diagram of behaviours to carry out the activity of booking appointments. The conditionals imply other behaviours; for example, the first conditional in Figure 7 implies that the controlling process must initiate the behaviour of reading the contents of Knowledge-of-tasks and, if necessary, perceiving the patient's requirement for appointment time (see Figure 4). Thus, this procedure plan for booking of appointments describes the behaviours of the worksystem in terms of both the planning, control, perception and execution behaviours and the transformation of the medical case objects that constitute the generic sub-task of appointment-booking (see next Section). Figure 7 is only a small section of the procedure plan for booking of appointments; the full plan appears in Appendix 2.

The Procedure plans, inferred in the form of mental routine structures, supported the effective performance of generic sub-tasks. Specifically, medical reception task quality, in support of medical cases, was maintained by:

- providing quick responses in a domain with a very high frequency of autonomous events (patients arriving, incoming telephone calls);

- maintaining consistency which supported the rotation of the four receptionists between the two medical reception workstations; 
- supporting shared behaviour, such that if one workstation was left unattended because a receptionist was busy, the other receptionist on that shift could take over at the unattended workstation.

Maintaining medical reception task quality by means of procedure plans was sometimes associated with higher medical reception costs (supporting shared user behaviour) and sometimes lower costs (supporting rotation of receptionists).

\section{The Relationship between the Types of Plans}

The following case of an appointment being booked illustrates the relationship between the three types of plan shown in Figure 4, and indicates how they operated in combination to guide the worksystem's behaviour.

\section{INSERT FIGURE 8 ABOUT HERE}

At the beginning of the day, the controlling process of the medical reception worksystem reads the activity plan - which specifies that receptionist $\mathrm{R}$ should carry out booking appointments from the front-desk during the morning (Figure 8) - and sets the parameters of the perceiving, executing and planning processes appropriately.

Later, an autonomous event occurs associated with the domain: patient $\mathrm{P}$ telephones the surgery requiring an appointment. The controlling process then reads from the procedure plan for booking appointments (Figure 8) which guides control decisions to activate the following sequence of behaviours:

- perception: detecting the values of patient P's attributes and updating knowledge-oftasks with the following attribute values:

appointment-requirements-who: own $\operatorname{Dr}(\operatorname{Dr} X)$

appointment-requirements-when: today

problem type: not emergency

- planning: selecting and (mentally) marking a possible appointment slot in the task plan (i.e. the appointment book): $\operatorname{Dr} X$, time $t$

- execution: offering the selected appointment to patient P, i.e. attempt to transform P's attribute values to:

appointment-practitioner: $\operatorname{Dr} X$

appointment-time: time $t$

- perception: updating knowledge-of-tasks to register the acceptance of the appointment and patient P's name.

- planning: adding a representation of the agreed appointment to the task plan

- perception: confirming the appointment details with patient $P$. 
This description completes the PCMT-MR model, based on the protocol data of the observational study and the PCMT framework.

\subsection{Extension of PCMT Framework}

The investigation of medical reception has indicated that the differentiated plan types of task, activity, and procedure are generic with respect to the MR model. Abstracting away from medical reception, it is hypothesised that the three plan types are also generic at the level of the PCMT framework. They might be expected to occur in other domains of planning and control of multiple tasks. The PCMT framework, therefore has been extended to accommodate: task plans; activity plans; and procedure plans. The extension is illustrated in Figure 4, which identifies the purely PCMT components of the plan framework.

\subsection{Medical Reception Performance}

As reported in Section 3.2, the MR model consists of the medical reception domain of application and the medical reception multiple task planning and control worksystem. The relationship between the medical reception domain and the medical reception worksystem can be expressed as MR performance. MR performance comprises two factors. MR task quality expresses the support provided to the medical case histories, that is how well they are supported. For example, the effective performance for the subtask of preparation of repeat prescriptions, (which transforms the medical case object attribute of treatment support accuracy), was supported by each repeat prescription being issued within 24 hours of a request being made, that is, with acceptable expedition. MR user costs express the physical and mental costs to the receptionists and devices of the medical reception worksystem in carrying out medical reception work. For example, to provide quick accurate responses to requests for appointments, in a domain with a very high frequency of appointment requests, either from patients arriving at the surgery, or from incoming calls, the mental costs to the receptionists were high. They needed to memorise, that is create a structural representation of some of the information appearing in the appointment book (time and name of doctor of available appointments), and process these appointment representations.

Both MR task quality and MR user costs for some medical reception worksystem can be described as desirable (acceptable) or undesirable (unacceptable). If MR task quality and MR user costs are as desired, then MR performance is acceptable (and there is no MR design problem). For example, the task quality of repeat prescriptions being prepared and appointments being booked was ensured, without increasing user costs, by having two receptionists at the surgery instead of one receptionist. The first receptionist could 
deal with the preparing of the repeat prescriptions, while the second could deal with the appointment-booking. MR performance is thus considered acceptable. However, if MR task quality and MR user costs are not as desired, then MR performance is not acceptable and there is a design problem. For example, in the mornings at the surgery, the number of incoming telephone calls, and patients arriving at the surgery can be very high. The front desk receptionist must deal with patients arriving at the surgery (to see the doctor, to book an appointment, to request a test result etc.) and requests for appointments through incoming telephone calls. If the receptionist is booking an appointment at the hatch, then they cannot answer the telephone, so another person at the surgery will answer the telephone. As each line is answered, if the caller requires an appointment, then they will be put on hold until the front desk receptionist can deal with them. There can be up to eight callers waiting to book appointments by telephone, and people waiting at the surgery to book appointments, and to make other requests of the receptionists. As the number of patients waiting increases, the MR task quality for the support of each medical case decreases, and the costs to the receptionists increase with the stress and confusion. MR performance would thus be considered unacceptable. The solution to the design problem would be the specification of the structures and behaviours of a medical reception worksystem whose MR performance would be acceptable.

Thus, the medical reception domain and worksystem are of necessity parts of the MR model, because both are required to express MR performance as MR task quality and MR user costs, and because MR design problems are expressed as unacceptable MR performance. Being commensurate in this way, allows the MR model to support the solution of MR design problems.

\section{THE USE OF THE MODEL IN SUPPORTING THE DESIGN OF MEDICAL RECEPTION WORKSYSTEMS}

The PCMT-MR model as indicated above is intended to be a design-oriented model. That is, it is intended to express an applied problem in worksystem design in a manner that is appropriate for its solution. The research has not yet demonstrated that the PCMT-MR model does in fact support better design solutions for the planning and control of interactive medical reception worksystems. Such work has yet to be carried out. However, the research has gone some way towards showing how the model might be used to solve design problems associated with interactive system performance. Firstly, the research targets a particular specify-and-implement type of design practice which the model would be intended to support - that of structured design methods. This specify- 
and-implement type of design practice is where solutions to design problems are iteratively specified and implemented until a desired implementation is achieved. Structured design methods distinguish the specification of interactive systems from their implementation, provide notations and procedures to support design, and use software tools to carry out the procedures (see e.g. Sommerville 1989).

Secondly, the research has implemented a computer simulation of the PCMT-MR model as a 'concept demonstrator' for a design tool. This simulation is written in an objectoriented discrete event simulation language called MODSIM-II. This tool is intended to show in principle how a design-oriented model might assist designers in generating and evaluating alternative medical reception worksystem configurations, by supporting the manipulation of planning and control structures and by predicting their effects on performance. By demonstrating and invesigating how the PCMT-MR model might support designers, this work aims to establish more firmly the desirable content, format and role of design-oriented models. Full details of the simulation will be reported elsewhere (Whitefield et al, manuscript in preparation). Suffice it to say here that all the components of the PCMT-MR model, including the domain and the worksystem, have been implemented in the simulation.

Thirdly, the research has mapped the concepts of the PCMT framework (and hence of the PCMT-MR model and the design tool) to the concepts used in structured design methods (the targeted type of design practice). Clear proposals exist, therefore, of how to incorporate the model into the design methods and how to represent its components for the designers. This would enhance this type of design practice through the reduction of the number of costly specify-implement cycles, by informing the specification process and introducing relevant knowledge at an early stage of design. Again, the full details of this mapping will be reported elsewhere (Whitefield et al, manuscript in preparation).

Within this scheme for the use of the model in design, it is possible to illustrate, with a simple example, how it might be used. Suppose the performance of the medical reception worksystem described in Section 3 was considered unacceptable to the doctors operating the medical practice. Suppose also that computerisation, and so the design of the worksystem interface, was considered one way of increasing MR performance and so making it acceptable. The design problem might be expressed as increasing MR performance relative to the non-computerised worksystem, either by increasing the task quality of medical case support or by reducing medical receptionists' costs or both. 
The PCMT-MR model would inform the designer of the identified plans and plan types. The designer would then need to consider whether and how the identified plans would be included in, and supported by, the new design of the worksystem. For example, the designer might consider two alternative ways to implement a procedure plan for appointment-booking. One way might involve, as the default presentation on the display screen, only the free appointment slots for each doctor; the filled appointments would be shown only when the medical receptionist requested them by some appropriate behaviour at the interface. The other way might involve, as the default presentation, all appointment slots for each doctor, both filled and free; in this case fewer free appointments will be shown on screen at any time, and the medical receptionist may need to request further appointments to be shown by some appropriate behaviour at the interface.

It would then be possible to use a design tool based on the PCMT-MR model to predict the performance of each of these proposed worksystem designs, and so prescribe a design solution, for different domains of application (i.e. for different numbers of doctors, patients, patterns of appointment requests, and so on). The (hypothetical) outcome might be as follows.

Both designs lead to the same task quality, and so meet that requirement of the design problem. That is, both designs can display on screen all the free appointments and these can therefore be offered to the patient for them to select the most suitable. Thus, the value of the medical case object attribute suitability of appointment for the patient will be transformed to suitable in both cases, and task quality will be acceptable. To transform the attribute suitability of appointment for the patient, the attribute values of appointment-time and appointment-medical practitioner of the patient sub-object must be transformed. The values of these attributes must then be compared with the attribute values of appointment requirements: who and appointment requirements: when of the patient sub-object, to determine the value of the suitability of the appointment for the patient attribute.

But there is a difference in the resource costs of the two designs. These costs vary as a function of the proportion of appointment requests which involve a change in the time of a previously booked appointment. When this proportion is low, the performance of the first worksystem design (showing only the free appointments) is more effective. The reason is because most patients want a new appointment, and this design presents the maximum number of free slots on screen at one time to offer to the patient. The result is low user costs for the worksystem because the receptionist has to perform the fewest number of planning behaviours to find a suitable appointment. Relevant planning 
behaviours here include: reading the knowledge-of-tasks representation to identify the appointment requirements: who and appointment requirements: when of the patient sub-object; reading the task plan representation (displayed on the screen) to identify the availability of the medical practitioner objects (i.e. free appointments); manipulating the task plan representation to display other free appointments; and matching free appointments to the patient's requirements. On occasions when the filled appointments do need to be shown (i.e. when a patient requests a change in the time of a previously booked appointment), the receptionist will have to perform more planning behaviours to display the filled appointments and their resource costs will therefore increase.

When the proportion of appointment change requests is high, the performance of the second worksystem design (showing both filled and free appointments) is more effective. The reason is because information about filled appointments is always present, which means the receptionist has to perform the fewest number of planning behaviours to change an appointment time, which leads to low resource costs for the worksystem. But for new appointment requests, the worksystem will need to perform more planning behaviours to find a suitable appointment (because fewer free appointments are shown at any time) which will increase the resource costs for the worksystem.

The overall performance of the two designs therefore differs, and the designer could make an appropriate decision, based on information about the particular domain, which would contribute to the solution of the design problem.

This example is intended to illustrate how the PCMT-MR model, in the form of a software design tool, might provide designers of interactive computer support with predictive information about worksystem performance and so prescriptive solutions to worksystem design problems. Such a design tool is not currently available. However, the construction of a tool and its application, both based on the the PCMT-MR model, are both in principle realisable.

\section{CONCLUDING COMMENTS}

This paper has reported an investigation of the planning and control of multiple task work in the domain of medical reception. The outcomes of the investigation can be summarised as:

- protocol data concerning medical reception

- a model of medical reception consisting of a worksystem and its domain of application

- an extension of the framework for the planning and control of multiple task work to include differentiated plan types 
- an illustration of the application of the model to solve a design problem associated with the performance of an interactive medical reception planning and control worksystem.

The current status of the outcomes of the investigation must be considered preliminary at this time. Data were collected concerning only one medical reception practice. Observation of additional medical practices would be necessary to verify and validate the model of medical reception. Further development of the model would seek to identify and conceptualise new phenomena and to refine the existing concepts. For the domain of application, development would provide a richer description of the object attributes, their required transformations and the relationships between those transformations. For the interactive worksystem, development would provide a richer description of planning and control structures. For performance, development would provide a wider and deeper description of resource costs incurred by the worksystems's planning and control behaviours and the task quality associated with the multiple task work carried out in the domain. Likewise, further studies of planning and control in domains other than secretarial office administration and medical reception would be necessary to confirm the framework for the planning and control of multiple task work. An investigation to this end has already been conducted into a third domain - that of solicitors' work (the data have been analysed, but not yet reported). Lastly, the computer simulation of the medical reception model needs to be developed into a software tool to support the solution of design problems associated with the performance of interactive medical reception worksystems.

Although its outcomes are preliminary at this time, the investigation reported here has nevertheless been able to present a complete illustration of its approach and so of its novelty. The research has attempted to extend the Broadbent tradition concerning the relation between theory and application in cognitive psychology. The research has attempted to meet all three requirements for developing the tradition identified earlier in Section 1.1. First, a technical expression for the applied problem has been proposed (as well as for the cognitive theory). The design problem (and also its design solution), associated with interactive medical reception worksystems, has been expressed as MR performance, the relationship between the medical reception domain and the medical reception worksystem - the two components of the theory (i.e. the model of medical reception). Second, and as required by the expression of the applied problem and the support for its solution, the scope of the theory is greater than just the human cognitive behaviours associated with the applied problem. Thus, the model of medical reception proposed here includes human as well as non-human (device) behaviours of the worksystem. It also includes an independent expression of the medical reception work 
carried out by the worksystem in its domain of application. Together, the worksystem and the domain of application can be used to construct an expression of MR performance, that is how well the medical reception worksystem carries out its medical reception work. Third, the form and granularity of the theory is commensurate with the applied problem (and so its solution). If the design problem is expressed as MR performance (at some desired level), then the model of the medical reception worksystem and its domain of application are of necessity commensurate with the problem, since MR performance is an expression of their relationship. Embodying the model of medical reception in a software tool will make it likewise commensurate in form for supporting the solution of design problems.

Attempting to meet these requirements is more likely to produce a theory which supports the solution of applied problems, or in Broadbent's terms a theory which works. We hope Donald would have approved 


\section{ACKNOWLEDGEMENT}

The work reported herein was supported by the Joint Councils Initiative in Cognitive Science/HCI, grant no: SPG 8825634.

\section{REFERENCES}

Ambros-Ingerson, J.A. 1986. Relationships between planning and execution. Quarterly Newsletter of the Society for the Study of Artificial Intelligence and Simulation of Behaviour, 57, 11-14.

Broadbent, D.E. (1971a) Relation between theory and application in psychology. Dorchester: Dorset Press.

Broadbent, D.E. (1971b) Decision and Stress. London: Academic Press.

Dowell, J. and Long, J. (1989) Towards a conception for an engineering discipline of human factors. Ergonomics, 32, , 1513-1536.

Drury, M. (1981) The medical secretary's and receptionist's handbook 4th ed. London: Bailliere Tindall.

Hayes-Roth, B. (1985). A blackboard architecture for control. Artificial Intelligence. 26, 251321.

Hollnagel, E., Mancini, G. and Woods, D. (1988). Cognitive Engineering in Complex Dynamic Worlds. London: Academic Press.

Jeffreys, M. and Sachs, H. (1983). Rethinking General Practice: Dilemmas in Primary Health Care. London: Tavistock.

Larkin, J.H. (1989) Display-based problem solving. In D.Klahr and K.Kotovsky (Eds) Complex Information Processing: The Impact of Herbert A. Simon. Hillsdale, NJ: Lawrence Erlbaum..

Newell, A and Simon, H. (1972) Human Problem Solving. Prentice-Hall.

Payne, S.J. (1991) Display-based action at the user interface. International Journal of ManMachine Studies, 35, 275-289.

Sommerville, I. (1989) Software Engineering 3rd ed. Wokingham: Addison Wesley.

Smith, M.W., Hill, B., Long, J.B. and Whitefield, A.D. (1992a) The Planning and Control of Multiple Task Work: a Study of Secretarial Office Administration. In Proceedings of the Second Interdisciplinary Workshop on Mental Models. Cambridge, 74-83, in press.

Smith, M.W., Hill, B., Long, J.B. and Whitefield, A.D. (1992b) Modelling the Relationship Between Planning, Control, Perception and Execution Behaviours in Interactive 
Worksystems. In D.Diaper, M.Harrison and A.Monk (Eds) People and Computers VII; Proceedings of HCI '92. Cambridge University Press.

Smith, M.W., Hill, B., Long, J.B. and Whitefield, A.D. (1993) A Design-Oriented Framework of the Planning and Control of Multiple Task Work. Submitted for publication.

Suchman, L.A. (1987) Plans and situated actions. Cambridge University Press.

Young R. and Simon T., 1987. Planning in the context of Human-Computer Interaction. In D.Diaper and R.Winder (Eds) People and Computers III. Cambridge University Press. 
Figure 2 A plan view of the medical reception worksystem

STREET ENTRANCE

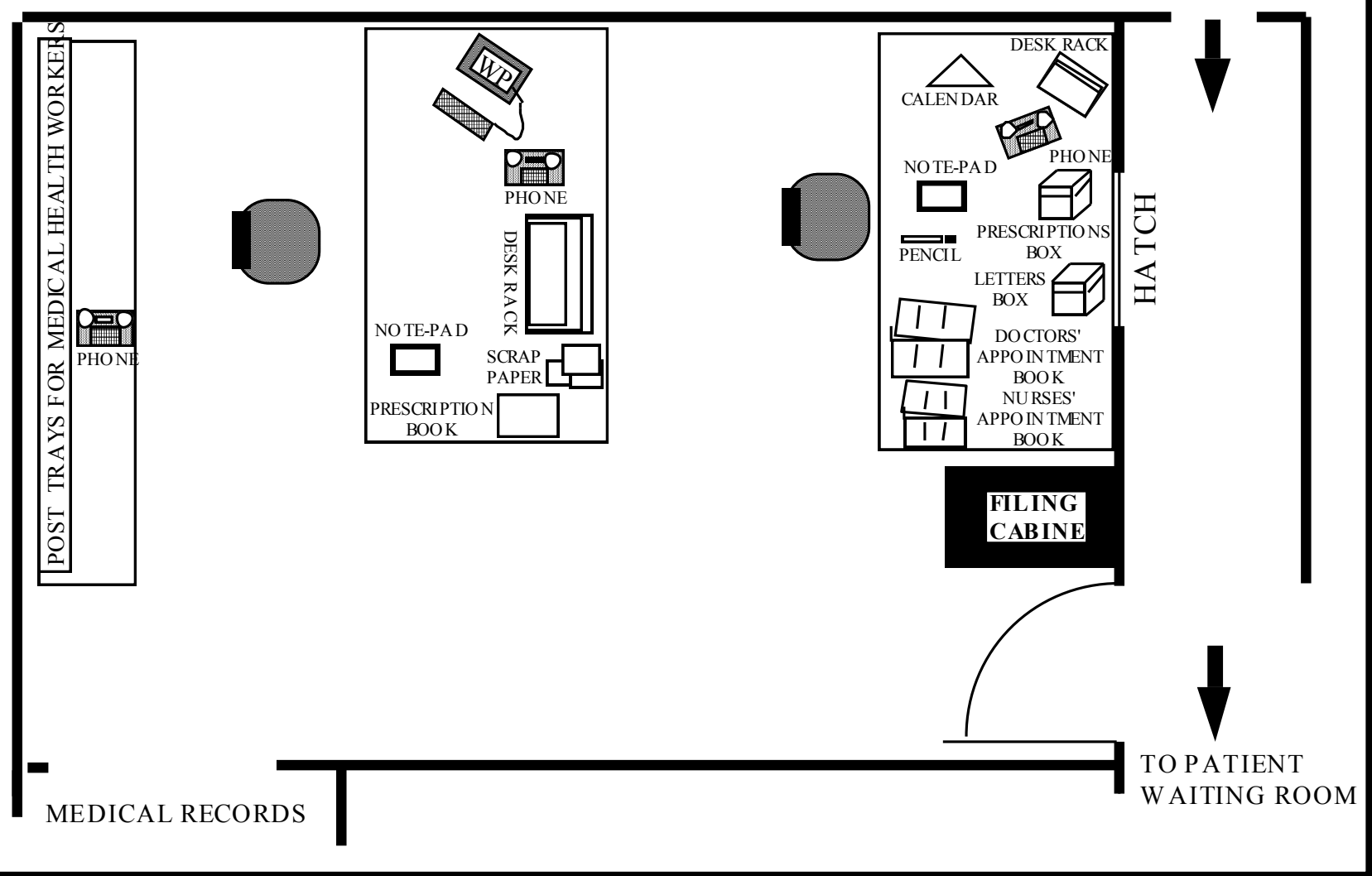


Figure 3: Extract from the protocol data

Telephone 1 flash

1 Receptionist1 PICK UP receiveratelephone1

1 Receptionist? (over) telephone SAY: Can I help?

2 Receptionist1 PICK UP green card (?baby registration card of P2) from hatch

2 Receptionisti READ green card

2 Receptionist1 PUT green card on desk-top

1 ReceptionistI (over) telephone SAY: No I'm sorry I've got nothing

Nurse look at Nurse-appointment-book

1 Receptionist (over) telephone SAY: Did you say it's an eye infection

1 Receptionist? (over) telephone SAY: Right. I've got literally nothing

1 Receptionist (over) telephone SAY: All I can offer you is 11:45 this morning an emergency appointment

Receptionist2 SEARCH prescription-box

1 Receptionist] (over) telephone SAY: Or 10 past 10 with Dr J tomorrow morning

Receptionist2 TAKE OUT prescription

1 Receptionist 1 (over) telephone SAY: OK your name again?

1 Receptionist1 WRITE in appointment-boolk

1 Receptionist1 (over) telephone SAY: OK: 11:45 Dr I

1 Receptionist1 (over) telephone SAY: Thank you bye

1 Receptionist1 REPLACE receiver-telephone1

2 Receptionist1 SAY: (to hatch) Have you not got a card when you registered the baby with the Shire Hall?

(For an explanation of the numbers see text)

KEY

\begin{tabular}{|l|l|}
\hline Orrtined text & physical device streucture of IWS \\
\hline UPPERCASE TEXT & BEHAVIOUR of the worksystem \\
\hline Underlined text & physical domain object \\
\hline
\end{tabular}


page 34 
Table 1 Generic sub-tasks

\begin{tabular}{|c|}
\hline appointment-booking \\
\hline repeat prescription preparation \\
\hline new patient registration \\
\hline medical notes for medical practitioners preparation (and updating) \\
\hline patient test results notification \\
\hline mail handling \\
\hline
\end{tabular}


Tables 2a-2c - Appointment-booking

Table 2a: Medical Case Object

\begin{tabular}{|l|l|l|l|}
\hline Attribute & Initial value & transform & Final value \\
\hline $\begin{array}{l}\text { suitability of appointment } \\
\text { for patient }\end{array}$ & not known & $-------->$ & suitable \\
\hline $\begin{array}{l}\text { suitability of appointment } \\
\text { for doctor }\end{array}$ & not known & $------->$ & suitable \\
\hline
\end{tabular}

Table 2b: Patient Object

\begin{tabular}{|c|c|c|c|}
\hline Attribute & Initial value & transform & Final value \\
\hline $\begin{array}{l}\text { appointment-requirements: } \\
\text { who }\left(^{*}\right)\end{array}$ & Dr $Y$ & & $\operatorname{Dr} Y$ \\
\hline $\begin{array}{l}\text { appointment-requirements: } \\
\text { when }\left({ }^{*}\right)\end{array}$ & Wednesday & & Wednesday \\
\hline problem type $\left(^{*}\right)$ & No emergency & & No emergency \\
\hline appointment-time & none & --------> & Wednesday \\
\hline $\begin{array}{l}\text { appointment-medical } \\
\text { practitioner }\end{array}$ & none & --------> & Dr Y \\
\hline $\begin{array}{l}\text { knowledge of appointment } \\
\text { availability }\end{array}$ & not informed & ---------> & informed \\
\hline
\end{tabular}

Table 2c: Medical Practitioner Object

\begin{tabular}{|l|l|l|l|}
\hline Attribute & Initial value & transform & Final value \\
\hline booking requirements $\left(^{*}\right)$ & squiggles open & & squiggles open \\
\hline availability $\left(^{*}\right)$ & $\begin{array}{l}\text { all } \\
\text { appointments }\end{array}$ & $\begin{array}{l}\text { all } \\
\text { appointments }\end{array}$ \\
\hline knowledge of appointment & not informed & --------> & informed \\
\hline
\end{tabular}

(For an explanation of a squiggle see Section 3.2.1 the Task plan) 
Tables 3a-3c - Repeat Prescription Preparation

Table 3a Medical Case Object

\begin{tabular}{|c|c|c|c|}
\hline Attribute & Initial value & transform & Final value \\
\hline treatment support accuracy & not known & ---------> & accurate \\
\hline \multicolumn{4}{|l|}{ Table 3b: Treatment Object } \\
\hline Attribute & Initial value & transform & Final value \\
\hline patient identity $\left({ }^{*}\right)$ & Mrs X & & Mrs X \\
\hline $\begin{array}{l}\text { medical practitioner identity } \\
\left({ }^{*}\right)\end{array}$ & Dr Y & & Dr Y \\
\hline informational content & none & --------> & $\begin{array}{l}\text { prescribed } \\
\text { treatment } X\end{array}$ \\
\hline authorisation & unauthorised & ---------> & authorised \\
\hline location & $\begin{array}{l}\text { with receptionist } \\
\text { with Dr Y } \\
\text { at reception }\end{array}$ & $\begin{array}{l}-\cdots--->> \\
-\cdots---> \\
-\cdots--->>\end{array}$ & $\begin{array}{l}\text { with Dr Y } \\
\text { with receptionist } \\
\text { with Mrs X } \\
\end{array}$ \\
\hline \multicolumn{4}{|c|}{ Table 3c: Medical Practitioner Object } \\
\hline Attribute & Initial value & transform & Final value \\
\hline intended treatment $\left(^{*}\right)$ & $\begin{array}{l}\text { prescribed } \\
\text { treatment } X\end{array}$ & & $\begin{array}{l}\text { prescribed } \\
\text { treatment } X\end{array}$ \\
\hline
\end{tabular}

Tables 4a-4c - Medical Notes Preparation and updating

Table 4a Medical Case Object

\begin{tabular}{|l|l|l|l|}
\hline Attribute & Initial value & transform & Final value \\
\hline Diagnosis support accuracy & not known & -------> & accurate \\
\hline
\end{tabular}

Table 4b: Diagnosis Object

\begin{tabular}{|l|l|l|l|}
\hline Attribute & Initial value & transform & Final value \\
\hline Patient identity $\left(^{*}\right)$ & Mrs X & & Mrs X \\
\hline $\begin{array}{l}\text { Medical practitioner identity } \\
\left({ }^{*}\right)\end{array}$ & Dr Y & Dr Y \\
\hline informational content & Mrs X details & $-------->$ & Mrs X details \\
\hline location & $\begin{array}{l}\text { in filing room } \\
\text { at reception }\end{array}$ & $\begin{array}{l}---------> \\
-------->\end{array}$ & $\begin{array}{l}\text { at reception } \\
\text { in Dr Y's tray }\end{array}$ \\
\hline
\end{tabular}

Table 4c: Patient object

\begin{tabular}{|l|l|l|l|}
\hline Attribute & Initial value & transform & Final value \\
\hline intended treatment $\left(^{*}\right)$ & $\begin{array}{l}\text { prescribed } \\
\text { treatment } \mathrm{X}\end{array}$ & & $\begin{array}{l}\text { prescribed } \\
\text { treatment } \mathrm{X}\end{array}$ \\
\hline appointment-time & Wednesday & & Wednesday \\
\hline $\begin{array}{l}\text { appointment-medical } \\
\text { practitioner }\end{array}$ & Dr Y & Dr Y \\
\hline
\end{tabular}


Table 5 Activities

\begin{tabular}{|c|}
\hline booking appointments \\
\hline preparing repeat prescriptions \\
\hline registering new patients \\
\hline preparing (and updating) medical notes for medical practitioners \\
\hline notifying patients of test results \\
\hline dealing with mail \\
\hline
\end{tabular}


Figure 5 The Appointment Book for the Doctors - a Partial Task Plan

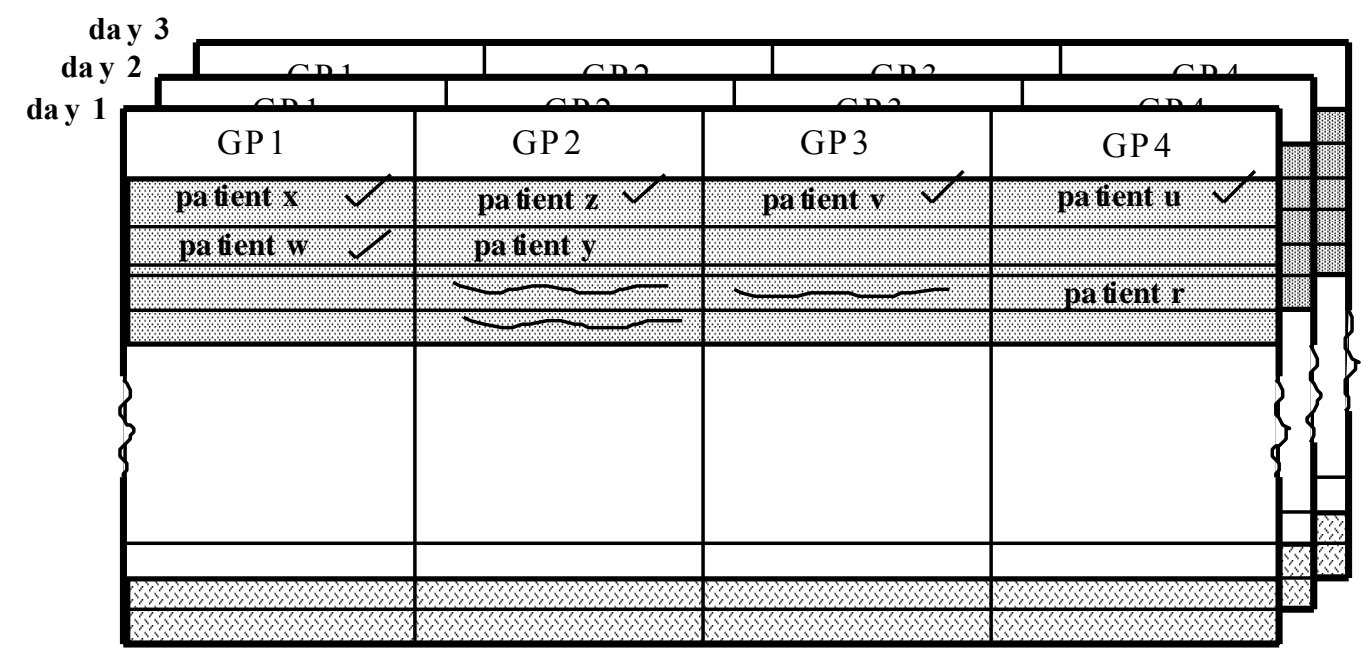

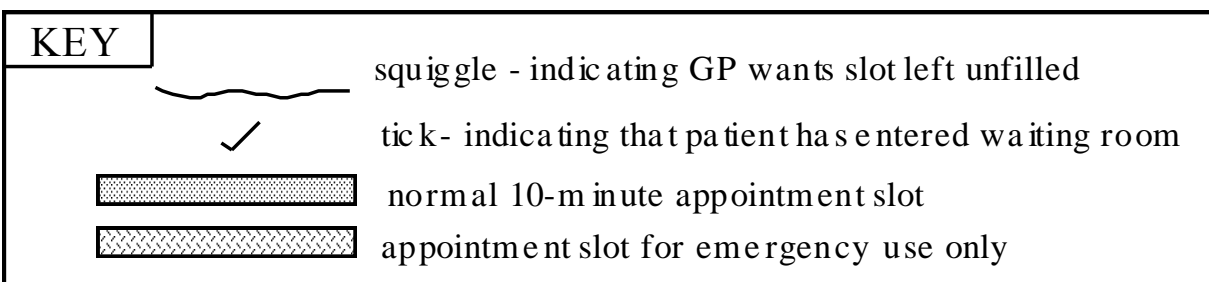


Figure 6 The Activity Plan

\begin{tabular}{|c|c|}
\hline BACK DESK & FRONT DESK \\
dealing with mail & morning \\
updating medical record envelopes & booking appointments \\
preparing repeat pres criptions & (requests, >10.00am) \\
afternoon & afternoon \\
dealing with mail & booking appointments \\
updating medical record envelopes & \\
preparing repeat pres criptions & \\
\hline
\end{tabular}


Figure 7 Part of a flow chart for the procedure plan for booking appointments

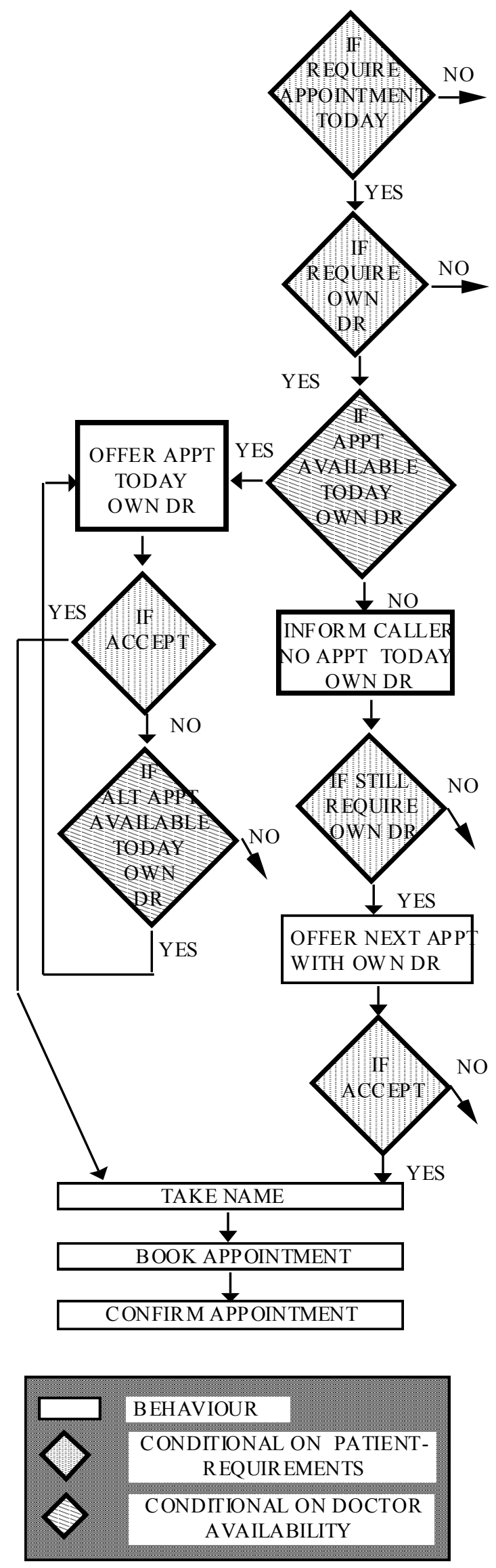


Figure 8 The relationship between plans

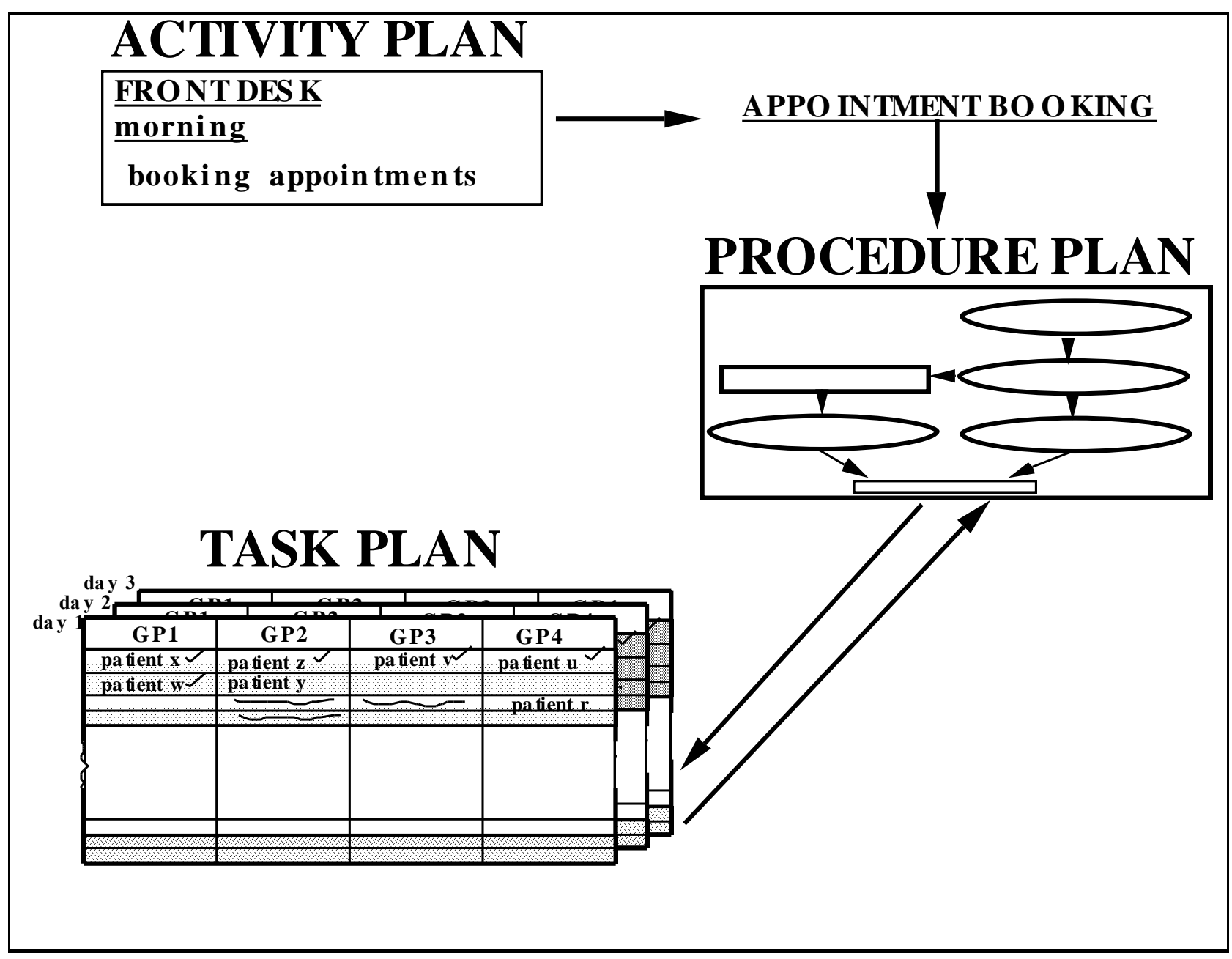




\section{APPENDIX 1}

Procedural descriptions of the activities carried out in medical reception.

\section{Preparing Repeat Prescriptions}

There are four ways for a patient to request a repeat prescription.

- Telephone the surgery and give the receptionist their name and drug required. This information is written on a piece of paper.

- Come into the surgery with their repeat prescription card (yellow card) and give it to the receptionist. The yellow card has the patients' name, drug, strength, number and total on the front. On the back of the card is the date when a prescription is issued and the drug number.

- Come into the surgery and request the drug but without a repeat prescription card. The patient will either have the request written down on a piece of paper already, fill in a repeat prescription request form (located on the hall table of the surgery), or give the request verbally to the front desk receptionist. The receptionist on the front desk will then give the requests to the receptionist on the back desk.

- Write to the surgery. The letter will be addressed to the Doctor concerned, but the receptionist will deal with the repeat prescription request, and then give the new prescription to the Doctor for signing with the letter from the patient attached.

The receptionist on the front desk will transfer any repeat prescription telephone requests to the back desk receptionist, if the appointment-booking is too busy on the front desk. The back desk receptionist takes all the requests to the filing room and accesses the notes which are contained in the medical record envelopes (MRE) for each of the requests. The receptionist then returns to the back desk with the Medical record envelopes for each patient. The receptionist boots up the computer database. The receptionist picks up one of the MRE's. The receptionist inputs the name and Date of Birth on the MRE into the database. The file is found on the computer and the receptionist then checks to see if the repeat prescription order has been entered on the computer. If the repeat prescription is on the database, the receptionist can print out the repeat prescription from the computer. If it is not, then the receptionist must check the information of the request with that on the MRE and then write out the prescription by hand. When a prescription has been produced, the receptionist must record this information on the MRE (and on the computer, if it is on the computer). Then, the prescription is put on the relevant Dr's tray with the MRE for them to sign. When the prescription has been signed, it is returned to the receptionists, who put the signed prescription in the prescription box on the front desk, ready for collection by the patient. 


\section{Digressions}

- If the repeat prescription is not on the computer, then the receptionist must inform the 'database compiler' by writing on a special form the name and drug of the patient, so this information can be input to the database. (The receptionists are not supposed to input the information themselves.

- If the repeat prescription is not on the computer, then the receptionist may need to look up the drug in MIMMS (drug reference book), as sometimes it is difficult to read the name of the drug and prescribing details on the yellow card and MRE. If it is on the database, it will not be a problem.

- If the drug being requested is not a regular repeat prescription drug, then the receptionist will have to write out the prescription as this drug will not be on the computer. An example would be a drug prescribed for a fungal infection, which a patient has recontracted and realises it is the same problem, so does not need to see the Dr, but just requires the same medication given before.

\section{Updating Medical Notes}

The Front desk receptionist makes a list on a piece of scrap paper of all people written in the appointment book for the next surgery session for each doctor.

The front desk receptionist hands this list to the back desk receptionist. The back desk receptionist takes the list to the filing room and accesses the notes which are contained in the medical record envelopes (MRE) for all the patients on the list. The receptionist then returns to the back desk with the Medical record envelopes for each patient. The receptionist boots up the computer database. The receptionist picks up one of the MREs. The receptionist inputs the name and Date of Birth on the MRE into the database. The file is found on the computer and the receptionist then checks the files contents with the contents of the MRE, and updates either the computer file or the MRE until both have the same information. The receptionist carries out the same process for all the MREs they have collected from the filing room. The receptionist then puts all the MREs and patient lists in the relevant Doctors tray.

As emergency slots for the next surgery session are filled in by the front desk receptionist, pieces of paper with the names of the patients taking those slots will be given to the back desk. The back desk receptionist must then go to the filing room and access the MRE's for those patients, and update the information using the same process as outlined above.

\section{Notifying patients of test results}

Patients either telephone or come into the surgery to request their test results. 
The receptionist requests information from the patient regarding their identity, when the test was carried out, and what the test was. (Results for differing tests take a different amount of time to be returned to the surgery.)

The receptionist will then go to the table at the back of the reception room. The receptionist will pick up the results book and look up the date the test was sent, and find the name of the patient on the specified date. The results book contains information about:

- the arrival of a test result at the surgery,

- authorisation of the result by the doctor (i.e. has the doctor seen the result)

- information to give to the patient regarding the result; i.e. if a doctor needs to be seen, if a drug needs to be prescribed, if the result is normal.

The receptionist will then check to see if the results have been returned and authorised in which case they will inform the patient of their result and the consequences for treatment.

If the results are not recorded in the book, and it is during the morning, then the receptionist will inform the enquirer to call back later. The result may have arrived in the morning post, but has not been seen by the Dr yet. This would not be available for collection until the afternoon. If it is not the morning, then the receptionist will inform the patient to return to the surgery, or ring the surgery on another day.

\section{Dealing with Mail}

The back desk receptionist deals with the mail coming into, and leaving the surgery. There are various types of mail, which are dealt with in different ways, as follows:

- The receptionist will put Letters to the doctor, which are personal, in the relevant doctors' tray.

- The receptionist will carry out the procedure for repeat prescriptions for any repeat prescription requests.

- The receptionist will mark any test result with a stamp which gives the date the result is received and has slots for the doctor to supply information regarding the follow up, i.e. is the test normal, does the patient need to see a doctor, etc.

- The receptionist puts letters from hospitals, other doctors etc. in the relevant doctors tray.

- The receptionist gives bills, receipts and payments to the group practice manager.

- The receptionist puts documents arriving from the Family Practitioner Committee in the relevant Doctors' or nurses' tray. 